\title{
The Economic Burden of Insulin-Related Hypoglycemia in Adults with Diabetes: An Analysis from the Perspective of the Italian Healthcare System
}

\author{
Witesh Parekh · Sophie E. Streeton · James Baker-Knight • Roberta Montagnoli • \\ Paolo Nicoziani · Giulio Marchesini
}

Received: February 23, 2018 / Published online: March 29, 2018

(C) The Author(s) 2018

\begin{abstract}
Introduction: The aim of this analysis was to estimate the cost of insulin-related hypoglycemia in adult patients with diabetes in Italy using the Local Impact of Hypoglycemia Tool (LIHT), and to explore the effect of different hypoglycemia rates on budget impact.

Methods: Direct costs and healthcare resource utilization were estimated for severe and nonsevere hypoglycemic episodes in Italy and applied to the population of adults with type 1 diabetes (T1DM) and type 2 diabetes (T2DM) and their corresponding hypoglycemia episode
\end{abstract}

Enhanced content To view enhanced content for this article go to https://doi.org/10.6084/m9.figshare. 6005075 .

Electronic supplementary material The online version of this article (https://doi.org/10.1007/s13300018-0418-0) contains supplementary material, which is available to authorized users.

W. Parekh

Novo Nordisk, Crawley, UK

S. E. Streeton · J. Baker-Knight

DRG Abacus, Bicester, Oxfordshire, UK

R. Montagnoli $(\varangle) \cdot$ P. Nicoziani

Novo Nordisk, Rome, Italy

e-mail: romo@novonordisk.com

G. Marchesini

Unit of Metabolic Diseases and Clinical Dietetics,

Alma Mater Studiorum University, Bologna, Italy rates (0.49 severe and 53.3 non-severe episodes per year for T1DM, and 0.09 severe and 9.3 nonsevere episodes per year for T2DM). Uncertainty around model inputs was explored through sensitivity and scenario analyses.

Results: The direct cost of insulin-related hypoglycemia in Italy is estimated at $€ 144.7$ million per year, with $€ 65$ million attributable to severe episodes and $€ 79.6$ million due to non-severe episodes. The total cost of hypoglycemia is approximately 1.7-fold higher for T2DM (€91.7 million) than for T1DM (€53 million). The cost of a hypoglycemic episode ranges from $€ 4.59$ for a non-severe event where additional self-monitoring of blood glucose (SMBG) testing is the only cost incurred, to $€ 5790.59$ for a severe event that also requires an ambulance, A\&E, hospitalization, and a visit to a diabetes specialist. A reduction in hypoglycemia event rates could result in substantial cost savings; for example, a $20 \%$ reduction in severe and non-severe hypoglycemia rates could result in a saving of $€ 47,769$ per general population of 100,000 people.

Conclusions: The LIHT highlights the substantial economic burden of insulin-related hypoglycemia in Italy, particularly with regards to non-severe hypoglycemia, an aspect of hypoglycemia that is often overlooked. This analysis may aid healthcare decision-making by allowing the costs of insulin therapies or diabetes self-management programs to be balanced with 
the savings provided by reductions in hypoglycemia.

Funding: Novo Nordisk, UK.

Keywords: Diabetes mellitus; Economic burden; Hypoglycemia; Insulin; Italy

\section{INTRODUCTION}

The prevalence of diabetes is increasing worldwide. In Italy, 3.18 million people aged 15 years and over were estimated to be suffering from diabetes in 2016 (6.1\% of the population for this age group) [1]. Type 1 diabetes (T1DM) and type 2 diabetes (T2DM) represent 5.4 and $91.9 \%$ of all diabetes cases in Italy, respectively, with the remaining $2.7 \%$ of cases made up of secondary diabetes $(2.1 \%)$ and 'other' types $(0.5 \%)$ [2].

Hypoglycemia, a common adverse effect of insulin therapy, is defined by the American Diabetes Association as blood glucose levels below an alert value of $70 \mathrm{mg} / \mathrm{dL}$ (with clinically significant hypoglycemia defined as a blood glucose level of $<54 \mathrm{mg} / \mathrm{dL}$ ) [3]. Hypoglycemia is defined as non-severe when the episode can be self-treated with glucose or a sweet drink or snack (15 g oral glucose according to Italian treatment guidelines for diabetes) [4]. Severe hypoglycemia requires third-party assistance and treatment with oral or intravenous glucose or intramuscular/subcutaneous injections of glucagon [4]. A severe episode may be treated by family or friends at home or work or may require assistance from emergency healthcare professionals (HCPs), in which case an ambulance, accident and emergency (A\&E) department and hospital treatment may be required. The Associazione Medici Diabetologi and Società Italiana di Diabetologia recommend selfmonitoring of blood glucose (SMBG) testing every $15 \mathrm{~min}$ after the onset of hypoglycemia until at least two normal values are measured in the absence of further treatment between the two measurements [4]. Patients may also consult their general practitioner (GP) or a diabetes specialist after a severe or non-severe hypoglycemic episode.
Hypoglycemia therefore represents a substantial economic burden to healthcare systems, with costs ranging from a few Euros for a non-severe episode, to very high costs for a severe episode requiring hospitalization of the patient [5]. Due to greater event frequency, the treatment of non-severe hypoglycemia may incur similar or greater annual costs than that of severe hypoglycemia [6, 7]. The aim of this study was to estimate the cost of insulin-related hypoglycemia in Italy using the Local Impact of Hypoglycemia Tool (LIHT), a model used previously for analyses in the UK [5], Denmark [6] and Spain [7]. In the current analysis we also explore the potential variation in budget impact arising from different hypoglycemia rates.

\section{METHODS}

The LIHT was developed for the UK and the methods have previously been described [5]. Briefly, the cost and utilization of specific healthcare resources are applied to a population of adults with T1DM and T2DM and their corresponding severe and non-severe hypoglycemia episode rates. In the current analysis, the LIHT was used to estimate the cost of hypoglycemia in Italy and the potential cost savings from a reduction in severe and nonsevere hypoglycemia rates.

\section{Patients and Hypoglycemia Rates}

The population of Italy, number of adults, and number of adults with diabetes were obtained from Italian National Institute of Statistics (ISTAT) data for 2016 [1, 8, 9]. At that time the total population of Italy was estimated to be $60,579,000$, with $86.3 \%$ aged $\geq 15$ years. Of those aged $\geq 15$ years, 3,182,000 people suffered from diabetes, corresponding to a prevalence of $6.1 \%$.

All patients with T1DM $(171,828)$ were assumed to be treated with insulin in this analysis. For patients with T2DM, the HYPOS-1 study, an Italian, questionnaire-based, retrospective study [10], was used to inform the proportion of insulin-treated patients. In HYPOS-1, 30.1\% of patients with T2DM were 
reported to be treated with insulin $(15.1 \%$ with oral antidiabetic drugs [OADs] plus insulin and $15.0 \%$ with insulin alone); the remaining patients were treated with OADs $(59.9 \%)$ and lifestyle intervention (10.0\%). Therefore, 880,202 patients with T2DM were assumed to be treated with insulin in the current analysis (Table 1).

Hypoglycemia rates were also taken from HYPOS-1; patients with T1DM were reported to experience an average of 0.49 severe and 53.3 non-severe episodes per year [11, 12], while patients with T2DM experienced an average of 0.09 severe and 9.3 non-severe episodes per year $[10,12]$. In line with Italian guidelines, severe hypoglycemia in the HYPOS-1 study was defined as an episode of hypoglycemia that led to unconsciousness or required intervention of a third person. Non-severe episodes were defined as the onset of one or more of the following symptoms, which resolved with the ingestion of food or a sugary drink: palpitations, tremors, sweating, difficulty concentrating, dizziness, hunger, blurred vision, confusion, or difficulty moving $[10,11]$.

\section{Resource Utilization}

The HYPOS-1 study informed utilization values for ambulance, A\&E, hospitalization, diabetes specialist visits, and GP visits [10-12]. As no data were identified on the percentages of severe episodes treated in different settings in Italy, these values were taken from a survey of people with diabetes in Germany, Spain, and the UK [13]. A weighted average across the three countries found that HCPs treated 33.3 and $43.2 \%$ of severe episodes experienced by people with T1DM and T2DM, respectively. For severe episodes treated by HCPs, it was assumed that the cost of glucose and/or glucagon was included in the ambulance, A\&E or hospitalization tariff to avoid double counting. Due to a lack of available utilization data, the cost of glucose and/or glucagon was assumed to be zero for severe episodes treated at work/home, and for non-severe episodes.

Patients use extra SMBG tests following a hypoglycemic episode to monitor their blood glucose more closely until it is stabilized. There was a lack of data for Italy on the number of extra SMBG tests used, so this information was taken from a survey-based study of 1404 patients with diabetes in the US, the UK, Germany, and France [14]. It was found that, on average, patients use 5.6 extra SMBG tests in the week following a non-severe episode. As no equivalent data were identified for severe episodes, the value for non-severe episodes was assumed to also apply to severe episodes. This is a conservative assumption, since patients are likely to monitor their blood glucose levels more closely following a severe episode than following a non-severe episode. The treatment pathway and resource utilization values applied in the model are shown in Fig. 1.

\section{Cost Inputs}

Costs for ambulance and hospitalization were taken from a study estimating the costs associated with emergency care and hospitalization

Table 1 Population in the model

\begin{tabular}{lr}
\hline Population & Number of people \\
\hline Total Italian population & $60,579,000$ \\
Adult Italian population (aged $\geq 15$ years) & $52,279,677$ \\
Adult Italian population with diabetes & $3,182,000$ \\
T1DM (5.4\% of diabetic population; all assumed to be insulin-treated) & 171,828 \\
T2DM (91.9\% of diabetic population) & $2,924,258$ \\
T2DM treated with insulin (30.1\% of T2DM population) & 880,202
\end{tabular}

T1DM type 1 diabetes mellitus, T2DM type 2 diabetes mellitus 


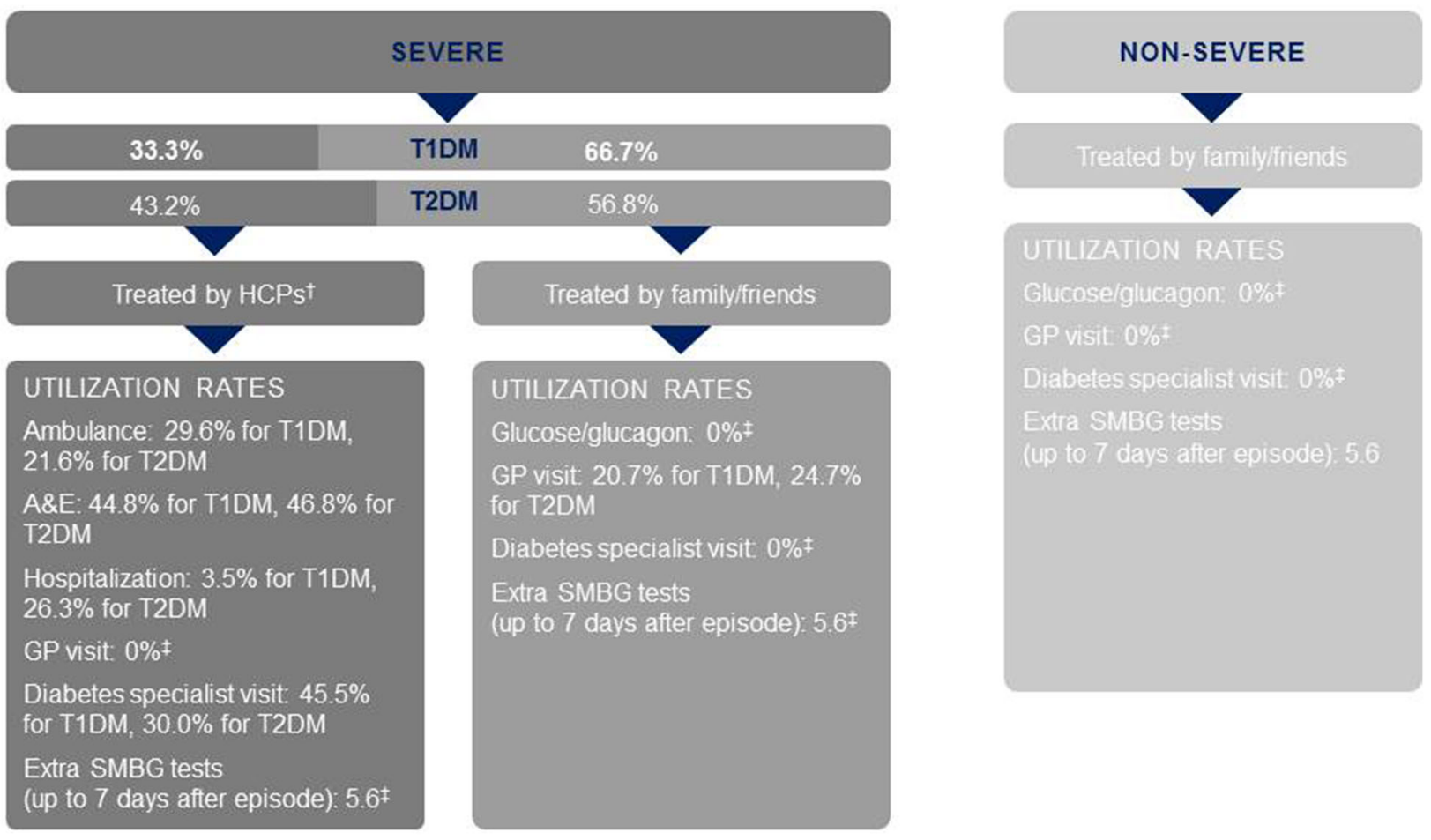

Fig. 1 Treatment pathway and resource utilization. $A \mho E$ accident and emergency, $G P$ general practitioner, $H C P$ healthcare professional, $S M B G$ self-monitoring of blood glucose, T1DM type 1 diabetes mellitus, T2DM type 2

for severe hypoglycemia in Italy [15]; hospitalization is a weighted average reimbursed cost per hospitalized patient with diabetes that is based on Diagnosis Related Group reimbursement methodology. Costs for A\&E and GP visits were taken from a cost analysis of the HYPOS-1 study [12]. The cost of a diabetes specialist visit was obtained from a study of the cost of management of severe hypoglycemia in Italy [15] and is in line with the cost of visits to other medical specialists published in Italian national tariffs [16]. The cost of an extra SMBG test was taken from a study of the cost-effectiveness of SMBG in patients with T2DM in France, Germany, Italy, and Spain [17] and was inflated to 2017 Euros using health price indices for Italy [18]. Cost inputs are shown in Table 2.

\section{Sensitivity Analyses}

Severe and non-severe hypoglycemia rates were varied by $\pm 10-50 \%$, both separately and in diabetes mellitus. Dagger $(\dagger)$ Patients treated by HCPs received treatment in the community (e.g., from a paramedic or medical practitioner) or in hospital. Double dagger $(\vdots)$ Assumed because no data were identified

combination, to explore the potential cost saving/increase associated with a reduction/increase in hypoglycemia rates. All model inputs were also varied by $\pm 20 \%$ to assess the level of uncertainty. Finally, the diabetes prevalence was varied in order to further explore the impact of hypoglycemia on the Italian healthcare system.

This article does not contain any studies with human participants or animals performed by any of the authors.

\section{RESULTS}

The LIHT highlights the cost burden of hypoglycemia in Italy for insulin-treated adults with diabetes. The direct cost of a hypoglycemic episode ranges from $€ 4.59$ to $€ 5,790.59$, where the minimum cost corresponds to a non-severe episode for which the only cost incurred is additional SMBG testing and the maximum cost 
Table 2 Minimum and maximum cost of a hypoglycemic episode for type 1 diabetes mellitus and type 2 diabetes mellitus in Italy

\begin{tabular}{llll}
\hline Resource & Cost inputs (unit cost [reference]) & Minimum cost of episode & Maximum cost of episode \\
\hline Ambulance & $€ 205.00[15]$ & - & $€ 205.00$ \\
A\&E & $€ 241.00[12]$ & - & $€ 241.00$ \\
Hospitalization & $€ 5317.00[15]$ & - & $€ 5317.00$ \\
GP visit & $€ 25.82[12]$ & - & - \\
Diabetes specialist visit & $€ 23.00[15]$ & - & $€ 23.00$ \\
Extra SMBG tests & $€ 0.82[17,18]$ & $€ 4.59$ & $€ 4.59$ \\
Total & $\mathrm{n} / \mathrm{a}$ & $€ 4.59$ & $€ 5790.59$ \\
\hline
\end{tabular}

$A \mho E$ Accident and emergency, $G P$ general practitioner, $n / a$ not applicable, $S M B G$ self-monitoring of blood glucose

is for a severe episode that is treated in hospital and requires an ambulance, the A\&E service, and a diabetes specialist (Table 2). The variation in cost per hypoglycemic episode arises due to differences in healthcare resource use for individual patients. The estimated average cost of a severe episode is $€ 683.14$ for T2DM and $€ 129.77$ for T1DM, while the average cost of a non-severe episode is $€ 4.59$ for both T1DM and T2DM. A breakdown of average costs by healthcare resource is shown in Fig. 2.

The total direct cost of insulin-related hypoglycemia in Italy is estimated to be $€ 144.7$ million per year, of which $€ 65$ million is attributable to severe episodes and $€ 79.6$ million is attributable to non-severe episodes. The total cost for a hypothetical general population of 100,000 people was also calculated to facilitate the assessment of cost burden in a specific region or hospital; this is estimated to be $€ 238,843$, with $€ 107,370$ and $€ 131,473$ due to severe and non-severe episodes, respectively (Table 3). The total cost of hypoglycemia associated with T2DM is approximately $€ 91.7$ million, 1.7-fold higher than the cost associated with T1DM ( $€ 53$ million; Table 3$)$. This difference can be largely attributed to the approximately five-fold higher total cost of treating severe episodes in patients with T2DM than in those with T1DM (approximately $€ 54.1$ million vs. $€ 10.9$ million), which is incurred despite patients with T2DM experiencing fewer severe episodes per year $(79,218$ vs. 84,196 for those with T1DM).

A reduction in hypoglycemia may be achieved, for example, by switching a patient's current insulin treatment to an insulin with a better hypoglycemic profile. To explore the potential variation in budget impact arising from differences in hypoglycemia event rate, the rates of severe and non-severe episodes were varied by $\pm 10-50 \%$, both separately in a oneway sensitivity analysis (Electronic Supplementary Material [ESM] Table 1) and in combination in a two-way sensitivity analysis (ESM Table 2). The results show that reductions in the rates of hypoglycemia events result in substantial cost savings. For example, in a sample general population of 100,000 , a $30 \%$ reduction leads to an annual cost saving of $€ 32,211$ for severe episodes, $€ 39,442$ for non-severe episodes and $€ 71,653$ for both severe and non-severe episodes combined.

To assess the uncertainty surrounding the model inputs, we performed one-way sensitivity analyses in which all unit costs and utilization values were varied by $\pm 20 \%$. The most influential variables were associated with treatment setting for patients with T2DM (percentage of patients treated by HCPs and percentage of patients hospitalized for a severe episode) and with additional SMBG testing after non-severe episodes. The main drivers of the model are shown in Fig. 3. 


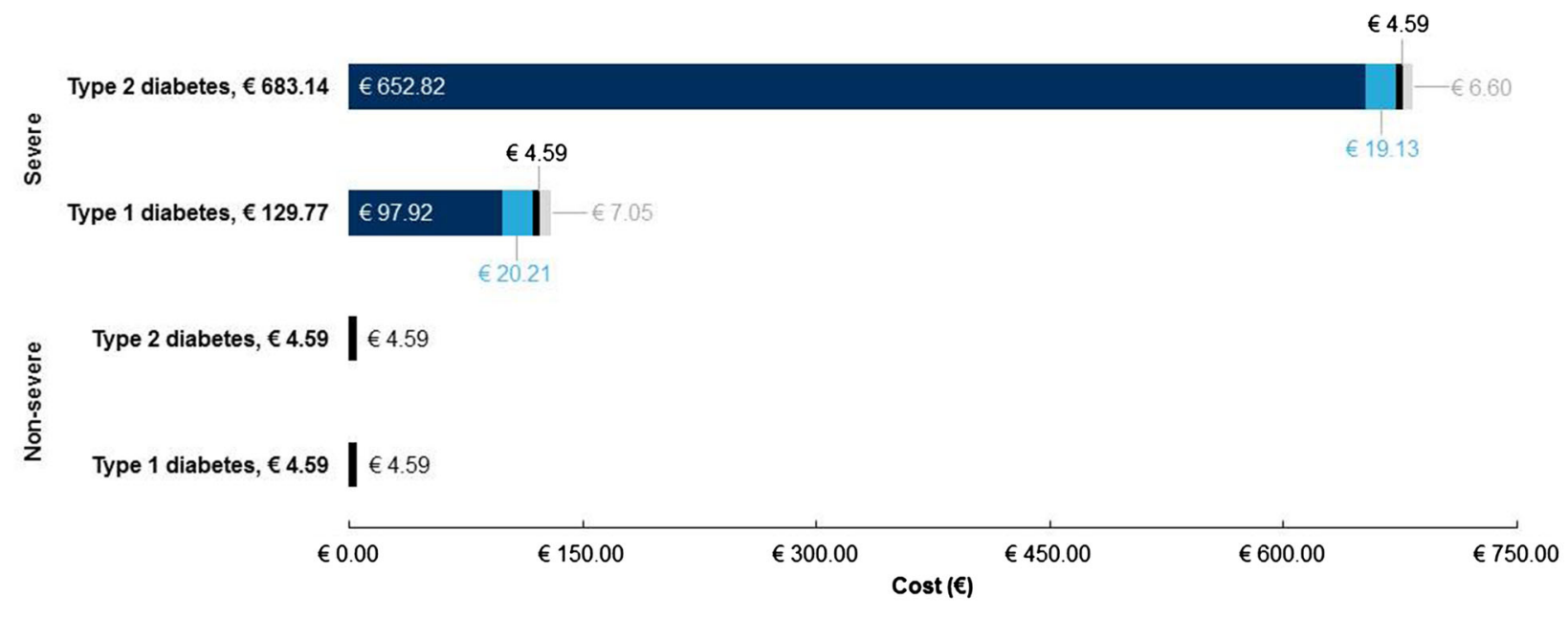

Hospital costs

Ambulance

Extra blood glucose tests

HCP consultations

Fig. 2 Cost breakdown by resource for an average severe and non-severe hypoglycemic episode. Hospital costs is the cost of $\mathrm{A} \& \mathrm{E}$ and hospitalization; HCP consultations is the cost of GP and diabetes specialist visits. HCP healthcare professional

Table 3 Cost of insulin-related hypoglycemia in Italy

\begin{tabular}{llll}
\hline Population $(n)$ & \multicolumn{2}{l}{ Severity of episode } & Total \\
\cline { 2 - 3 } & \multicolumn{1}{l}{ Severe } & Non-severe & \\
\hline Italian general population $(60,579,000)$ & & & \\
T1DM (171,828) & $€ 10,926,180$ & $€ 42,055,522$ & $€ 52,981,702$ \\
T2DM (2,924,258, of whom 880,202 are receiving insulin) & $€ 54,117,474$ & $€ 37,589,540$ & $€ 91,707,014$ \\
Total & $€ 65,043,654$ & $€ 79,645,062$ & $€ 144,688,715$ \\
Sample general population $(100,000)$ & & & \\
T1DM (284) & $€ 18,036$ & $€ 69,423$ & $€ 87,459$ \\
T2DM (4827, of whom 1453 are receiving insulin) & $€ 89,334$ & $€ 62,050$ & $€ 151,384$ \\
Total & $€ 107,370$ & $€ 131,473$ & $€ 238,843$ \\
\hline
\end{tabular}

$n$ number of patients, TIDM type 1 diabetes mellitus, T2DM type 2 diabetes mellitus

ISTAT data for 2016 show that the overall prevalence of diabetes in Italy varies between regions, ranging from $3.2 \%$ in the Autonomous Province of Trento in the north, to $7.9 \%$ in the southern region of Calabria [19]. A sensitivity analysis exploring the impact of diabetes prevalence on the cost of insulin-related hypoglycemia was therefore also performed (ESM Table 3). A disease prevalence of $8.0 \%$ in a sample general population of 100,000 is associated with a total cost of $€ 313,932$, which is an increase of $€ 75,089$ compared with the default model prevalence of $6.1 \%$. A prevalence of $3.0 \%$ is associated with a cost of $€ 117,724$ (reduction of $€ 121,119$ versus the default model).

Finally, alternative model inputs for glucose and/or glucagon utilization, and SMBG unit cost, were tested in scenario analyses (ESM Table 4). In the base case analysis the cost of 


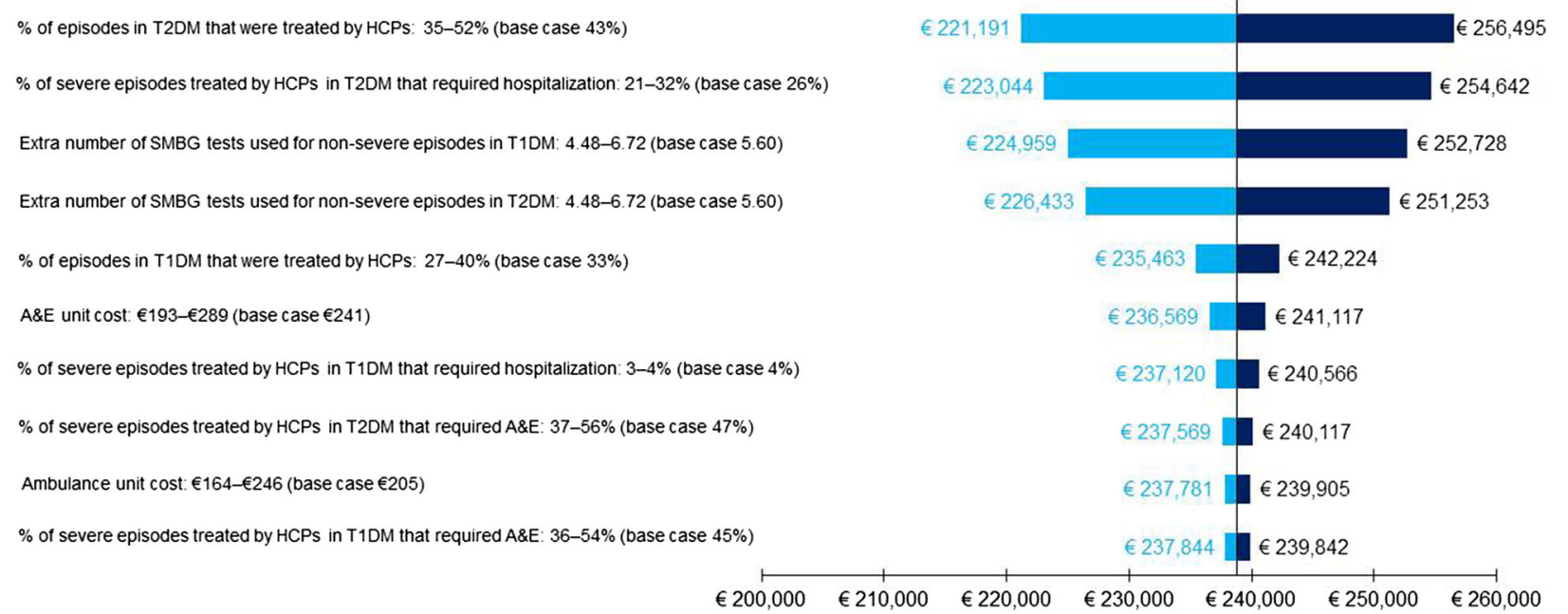

Fig. 3 Tornado diagram showing the main drivers of the model for a sample general population of 100,000. A\&E accident and emergency, HCP healthcare professional,

glucose and/or glucagon was conservatively assumed to be zero for severe hypoglycemic episodes treated at home or work as no data on the utilization of treatment were identified. The potential impact of this assumption was investigated by applying the average utilization and costs of the UK and Danish LIHT analyses [5, 6]; this had a small effect on the overall results, increasing the total cost of T1DM and T2DM by $€ 22,724$ and $€ 18,207$, respectively, from the default values. Applying alternative SMBG unit costs had a larger effect on the results, with the greatest impact observed when this cost was taken from a study in patients with T2DM that used data on SMBG unit cost from two regions in northern Italy [20].

\section{DISCUSSION}

In this study we highlight the cost to the Italian healthcare system of insulin-related hypoglycemia in adults with diabetes. The results show that, although frequently overlooked, non-severe hypoglycemia incurs the greatest cost, with an estimated total of $€ 79.6$ million per year (55\% of the total annual cost). The total cost of hypoglycemia associated with T2DM is approximately 1.7 -fold higher than that associated with T1DM.
$S M B G$ self-monitoring of blood glucose, TIDM type 1 diabetes mellitus, T2DM type 2 diabetes mellitus

The higher average cost of a severe episode for patients with T2DM compared with that for those with T1DM likely reflects the fact that, on average, people with T2DM have a shorter duration of diabetes and are therefore less familiar with hypoglycemia and the treatment required. These individuals may be more likely than those with T1DM to require medical assistance for a severe episode instead of receiving treatment from family or friends. This, along with the association of T2DM with older age (and therefore increased frailty) and the presence of comorbidities, is likely to increase the need for hospital admission. Indeed, for patients with diabetes experiencing severe hypoglycemia, T2DM was recently found to be associated with more frequent hospitalizations and longer length of hospital stay compared with T1DM [12].

In the sensitivity analyses, the proportion of patients with T2DM who received treatment from a HCP for a hypoglycemic episode, the proportion of patients with T2DM who were treated in hospital for a severe episode, and the number of extra SMBG tests used following non-severe episodes, had the largest effect on the overall results; these are therefore the inputs associated with the greatest uncertainty. Utilization of HCPs, A\&E, and hospital 
treatment were also identified as key drivers of the model. SMBG testing and the utilization of services such as HCPs and hospitals were also key model drivers in analyses performed using the LIHT in the UK [5], Denmark, [6] and Spain [7].

Hypoglycemia is a common adverse effect of insulin therapy and despite pharmacological and technological advances, event rates in clinical practice remain high [21]. Hypoglycemia is a key barrier to the intensification of insulin therapy; physicians acknowledge that many insulin-treated patients do not have adequate glycemic control, and 70\% would treat more aggressively were hypoglycemia not a concern [22]. Hypoglycemia risk can be reduced by switching a patient's current insulin treatment to insulin therapy with a better hypoglycemic profile; indeed, reductions of up to $50 \%$ have been observed upon switching from neutral protamine Hagedorn (NPH) insulin to insulin analogues [23, 24], or from older to newer insulin analogues [25, 26]. However, other factors also contribute to hypoglycemia rates. In particular, structured education and training programs in self-management of diabetes are now recognized as an important strategy for minimizing hypoglycemia risk [27]. The LIHT allows healthcare decision-makers to explore how introducing new insulin therapies or self-management programs could impact healthcare budgets by enabling them to balance these costs with the savings that can be realized when hypoglycemia rates are reduced. In the current study, sensitivity analyses exploring the impact of varying hypoglycemia rates show that reductions in severe and non-severe events result in substantial cost savings.

The costs of hypoglycemia in Italy are unlikely to be overestimated in this study because of several conservative assumptions. Firstly, only an adult, insulin-treated population is considered in the model. There are far fewer children than adults with diabetes in Italy, and it is difficult to speculate about the cost of hypoglycemia in these patients compared with adults. However, there will inevitably be additional costs of treating hypoglycemia for the 21,000 children aged $0-14$ years with diabetes in Italy [1]. The model also only considers insulin-related hypoglycemia; however, while hypoglycemia is most commonly associated with insulin treatment, it is also a complication of other glucose-lowering medications, such as sulfonylureas and glinides [28].

Secondly, the hypoglycemia rates used in the analysis are likely to be conservative. Rates of severe and non-severe hypoglycemia were taken from HYPOS-1 [10-12], a retrospective, questionnaire-based study that relied on patient recall. Hypoglycemic episodes are frequently under-reported by patients in real-world practice; indeed, a study of insulin-treated diabetes across seven European countries found that $65 \%$ of patients with T1DM and $50-59 \%$ of patients with T2DM rarely or never inform their GP or diabetes specialist about non-severe hypoglycemic events [29]. Patients may be reluctant to report hypoglycemia for several reasons, including implications for employment, the risk of losing their driving license, and the fear that they will be perceived to have poor disease control. The model also does not account for asymptomatic hypoglycemia, an aspect of diabetes associated with impaired awareness of hypoglycemia. Approximately $50 \%$ of patients with T1DM $[29,30]$ and $45 \%$ with T2DM $[29,31]$ were reported to experience impaired awareness of hypoglycemia in questionnaire-based studies.

Thirdly, indirect costs incurred when absence from work due to hypoglycemia results in lost productivity are not considered in the model, as they do not directly affect the clinician or budget holder. The financial impact on the patient and their family and friends is therefore an additional cost to society that is not accounted for in the current study.

Finally, while hypoglycemia is an acute complication of diabetes, it may also impose a long-term cost burden, for example when fear of hypoglycemia leads patients to reduce or omit an insulin dose, leading to sub-optimal glucose control and increased risk of long-term complications [32, 33]. Such long-term cost implications of hypoglycemia are not considered in the current analysis.

There are some limitations associated with the LIHT analysis due to a lack of resource utilization data for Italy. Firstly, data on the 
percentages of severe episodes treated in different settings (by family and friends at home/work or by HCPs in the community/hospital) were taken from a survey of people with severe hypoglycemia in Germany, Spain, and the UK [13]. Secondly, due to a lack of utilization data, glucose and/or glucagon costs are conservatively assumed to be zero for non-severe episodes and for severe episodes treated at home/work. When tested in a scenario analysis by applying the average utilization and costs from the UK and Danish LIHT analyses $[5,6]$, this assumption was found to have only a small impact on the overall results. For severe episodes treated in the community/hospital, glucose and/or glucagon costs are assumed to be included in the cost of ambulance, A\&E, or hospitalization tariff to avoid double counting. Finally, as Italian data on the number of extra SMBG tests used following hypoglycemic episodes were not available, a survey of patients with diabetes experiencing non-severe episodes in the US, UK, Germany, and France [14] was used. The values for non-severe episodes are assumed to also apply to severe episodes, a conservative assumption given that patients are likely to monitor their blood glucose levels more frequently following a severe episode than a non-severe episode. Given the potential for regional variation in SMBG unit costs in Italy [34], and the observed impact of extra SMBG testing in sensitivity analyses, alternative costs of SMBG testing were also explored in scenario analyses. The greatest impact on the results was seen when the SMBG unit cost reported by Afonso et al. [20] was applied; this study was not selected for the base case analysis as the SMBG unit cost reflects two northern Italian regions only.

\section{CONCLUSIONS}

This study highlights the substantial economic burden of insulin-related hypoglycemia for adults with diabetes in Italy. The LIHT is a useful model for estimating the costs of severe and non-severe hypoglycemic episodes for Italy as a whole, as well as for individual regions or hospitals. The tool may also aid healthcare decision-makers in their treatment choices by allowing the costs of insulin therapies or diabetes self-management programs to be balanced with the savings provided by reductions in hypoglycemia rates.

\section{ACKNOWLEDGEMENTS}

Funding. This study, article processing charges, and editorial assistance were funded by Novo Nordisk, Crawley, UK.

Editorial Assistance. Editorial support was provided by DRG Abacus (funded by Novo Nordisk).

Authorship. All authors had full access to all of the data in this study and take complete responsibility for the integrity of the data and accuracy of the data analysis. All named authors meet the International Committee of Medical Journal Editors (ICMJE) criteria for authorship for this manuscript, take responsibility for the integrity of the work as a whole, and have given their approval for this version to be published.

Disclosures. Witesh Parekh is an employee of Novo Nordisk, UK. Roberta Montagnoli is an employee of Novo Nordisk, Italy. Paolo Nicoziani is an employee of Novo Nordisk, Italy. Giulio Marchesini participates in advisory boards for Eli Lilly and Gilead, has received honoraria from Sanofi-Aventis, Merck Sharp \& Dohme and Novartis, and has been involved in clinical studies for Novo Nordisk, Boehringer Ingelheim, Sanofi-Aventis, Eli Lilly, Gilead, Genfit, Janssen, and AstraZeneca. Sophie Streeton is an employee of DRG Abacus. James BakerKnight is an employee of DRG Abacus.

Compliance with Ethics Guidelines. This article does not contain any studies with human participants or animals performed by any of the authors.

Data Availability. The datasets generated during and/or analyzed during the current 
study are available from the corresponding author on reasonable request.

Open Access. This article is distributed under the terms of the Creative Commons Attribution-NonCommercial 4.0 International License (http://creativecommons.org/licenses/ by-nc/4.0/), which permits any noncommercial use, distribution, and reproduction in any medium, provided you give appropriate credit to the original author(s) and the source, provide a link to the Creative Commons license, and indicate if changes were made.

\section{REFERENCES}

1. Italian National Institute of Statistics (ISTAT). Data table 2016. Health statistics: health conditions: health status: age class. Number of people aged 15 and over suffering from diabetes. http://dati.istat.it/ ?lang=en. Accessed November 2017.

2. Associazione Medici Diabetologi (AMD). AMD annals 2010: quality indicators in diabetes care in Italy. http://aemmedi.it/files/ANNALI-AMD/2010/ Annali\%202010\%20inglese.pdf. Accessed November 2017.

3. American Diabetes Association. Standards of medical care in diabetes-2017. Diabetes Care. 40[Suppl 1];S1-S135.

4. Associazione Medici Diabetologi (AMD) and Societa Italiana di Diabetologia (SID). Standard italiani per la cura del diabete mellito 2016. http://www. standarditaliani.it/skin/www.standarditaliani.it/pdf/ STANDARD_2016_June20.pdf

5. Parekh WA, Ashley D, Chubb B, Gillies H, Evans M. Approach to assessing the economic impact of insulin-related hypoglycaemia using the novel local impact of hypoglycaemia tool. Diabetic Med. 2015;32:1156-66.

6. Hoskins N, Tikkanen CK, Pedersen-Bjergaard U. The economic impact of insulin-related hypoglycemia in Denmark: an analysis using the Local Impact of Hypoglycemia Tool. J Med Econ. 2017:20(4): 363-370.

7. Parekh W, Hoskins N, Baker-Knight J, de Arellano AR, Raya PM. The economic burden of insulin-related hypoglycemia in Spain. Diabetes Ther. 2017;8(4):899-913.
8. Italian National Institute of Statistics (ISTAT). Demographic indicators 2016. http://www.istat.it/ en/archive/197555. Accessed November 2017.

9. Italian National Institute of Statistics (ISTAT). Data table 2016. Population and households: population: demographic indicators. Percentage of population aged 15 and over. http://dati.istat.it/?lang= en. Accessed November 2017.

10. Giorda CB, Ozzello A, Gentile S, et al. Incidence and correlates of hypoglycemia in type 2 diabetes. The Hypos-1 study. J Diabetes Metab. 2014;5:344.

11. Giorda CB, Ozzello A, Gentile S, et al. Incidence and risk factors for severe and symptomatic hypoglycemia in type 1 diabetes. Results of the HYPOS- 1 study. Acta Diabetol. 2015;52:845-53.

12. Giorda CB, Rossi MC, Ozzello O, et al. Healthcare resource use, direct and indirect costs of hypoglycemia in type 1 and type 2 diabetes, and nationwide projections. Results of the HYPOS-1 study. Nutr Metab Cardiovasc Dis. 2017;27:209-16.

13. Hammer M, Lammert M, Mejías SM, Kern W, Frier BM. Costs of managing severe hypoglycaemia in three European countries. J Med Econ. 2009;12:281-90.

14. Brod M, Christensen T, Thomsen TL, Bushnell DM. The impact of non-severe hypoglycemic events on work productivity and diabetes management. Value Health. 2011;14:665-71.

15. Veronese G, Marchesini G, Forlani G, et al. Costs associated with emergency care and hospitalization for severe hypoglycemia. Nutr Metab Cardiovasc Dis. 2016;26:345-51.

16. Italian Ministry of Health. Tariff for specialist outpatient care (Allegato 3). http://www.trovanorme. salute.gov.it/norme/renderPdf.spring?seriegu=SG\& datagu $=28 / 01 / 2013 \&$ redaz $=13$ A00528 \&artp $=3 \&$ art $=$ $1 \&$ subart=1\&subart $1=10 \&$ vers $=1 \& p r o g=001$. Accessed November 2017.

17. Tunis SL, Willis WD, Foos V. Self-monitoring of blood glucose (SMBG) in patients with type 2 diabetes on oral anti-diabetes drugs: cost-effectiveness in France, Germany, Italy, and Spain. Curr Med Res Opin. 2010;26:163-75.

18. European Central Bank. Health price indices for Italy, October 2017. https://www.ecb.europa.eu/ stats/ecb_statistics/escb/html/table.en.html?id=JDF_ ICP_COICOP_INX. Accessed November 2017.

19. Italian National Institute of Statistics (ISTAT). Data table 2016. Health statistics: health conditions: health status: regions and type of municipality. 
http://dati.istat.it/?lang=en. Accessed November 2017.

20. Afonso M, Ryan F, Pitcher A, Lew E. Evaluating drug cost per responder and number needed to treat associated with lixisenatide on top of glargine when compared to rapid-acting insulin intensification regimens on top of glargine, in patients with type 2 diabetes in the UK, Italy, and Spain. J Med Econ. 2017;20:633-9.

21. Khunti K, Alsifri S, Aronson R, et al. Rates and predictors of hypoglycaemia in 27585 people from 24 countries with insulin-treated type 1 and type 2 diabetes: the global HAT study. Diabetes Obes Metab. 2016;18:907-15.

22. Peyrot M, Barnett AH, Meneghini LF, SchummDraeger PM. Insulin adherence behaviours and barriers in the multinational global attitudes of patients and physicians in insulin therapy study. Diabetic Med. 2012;29:682-9.

23. Horvath K, Jeitler K, Berghold A, et al. Long-acting insulin analogues versus NPH insulin (human isophane insulin) for type 2 diabetes mellitus. Cochrane Database Syst Rev. 2007:CD005613.

24. Monami M, Marchionni N, Mannucci E. Long-acting insulin analogues vs. NPH human insulin in type 1 diabetes. A meta-analysis. Diabetes Obes Metab. 2009;11:372-8.

25. Lane W, Gerety G; Gumprecht J, Philis-Tsimikas A, Hansen CT, Nielsen TSS, Warren M. SWITCH 1: reduced hypoglycemia with insulin degludec (IDeg) versus insulin glargine (IGlar), both U100, in patients with T1D at high risk of hypoglycemia: a randomized, double-blind, crossover trial. Poster presented at the American Diabetes Association, 76th annual scientific sessions, 10-14 June 2016. New Orleans, LA, USA.

26. Wysham CB, Chaykin L, de la Rosa R, Handelsman Y, Troelsen LN, Kvist K, Norwood P. SWITCH 2: Reduced hypoglycemia with insulin degludec (IDeg) versus insulin glargine (IGlar), both U100, in patients with T2D at high risk of hypoglycemia: a randomized, double-blind, crossover trial. Poster presented at the American Diabetes Association, 76th annual scientific sessions, 10-14 June 2016. New Orleans, LA, USA.

27. Iqbal A, Heller SR. The role of structured education in the management of hypoglycaemia. Diabetologia. 2017:61(4):751-760.

28. Inzucchi SE, Bergenstal RM, Buse JB, et al. Management of hyperglycemia in type 2 diabetes, 2015: a patient-centered approach: update to a position statement of the American Diabetes Association and the European Association for the Study of Diabetes. Diabetes Care. 2015;38:140-9.

29. Ostenson CG, Geelhoed-Duijvestijn P, Lahtela J, Weitgasser R, Markert Jensen M, Pedersen-Bjergaard U. Self-reported non-severe hypoglycaemic events in Europe. Diabetic Med. 2014;31:92-101.

30. Pedersen-Bjergaard U, Pramming S, Thorsteinsson B. Recall of severe hypoglycaemia and self-estimated state of awareness in type 1 diabetes. Diabetes Metab Res Rev. 2003;19:232-40.

31. Akram K, Pedersen-Bjergaard U, Carstensen B, Borch-Johnsen K, Thorsteinsson B. Frequency and risk factors of severe hypoglycaemia in insulintreated Type 2 diabetes: a cross-sectional survey. Diabetic Med. 2006;23:750-6.

32. Leiter LA, Yale JF, Chiasson JL, Harris S, Kleinstiver P, Sauriol L. Assessment of the impact of fear of hypoglycemic episodes on glycemic and hypogycemia management. Can J Diabetes. 2005;29:186-92.

33. Wild D, von Maltzahn R, Brohan E, Christensen T, Clauson P, Gonder-Frederick L. A critical review of the literature on fear of hypoglycemia in diabetes: implications for diabetes management and patient education. Patient Educ Couns. 2007;68:10-5.

34. Italian National Agency for Regional Healthcare. Outpatient services: comparison of rates. http://www. agenas.it/prestazioni-specialistiche-ambulatorialiconfronto-tra-tariffe. Accessed November 2017. 